Editorial

\title{
Mobilizing patient data towards precision medicine
}

\section{Editorial}

In the digital age and under the pressure of healthcare contingencies, individual patient meta-data or digital identity ${ }^{1}$ has become a new form of currency. ${ }^{2}$ However medical scientists, clinicians and healthcare stakeholders still face a challenge regarding both data access and knowledge sharing capabilities so as to truly actionate precision Medicine. The first paradox is that we still dedicate more than $98 \%$ of our resources to generate relatively small amounts of novel data when Real Word offers yotabytes of useable external data, starting with the patient own legacy data. The second paradox is that individual patient information is still not integrated and accessible on a set of digital sources hence searchable system leading medical care professional to repeat the same queries. At a time we live a deep knowledge transformation with formidable initiatives, following the Human Genome Project, such as in Cancer with the International Cancer Genome Consortium ${ }^{3}$ (ICGC) or in Neurosciences with the Human Brain project ${ }^{4}$ in Europe or its US counterpart (BRAIN), ${ }^{5}$ the opportunity to change and Innovate by capitalizing on Big Data sources for Patient Care is real.

As we pursue and expand on our Translational Medicine driven transformation of patient care while delivering treatment and diagnosis Innovation, the combined mining of patient data with selected Open sources of Big Data, such as clinical genomics data, is indeed a sizeable opportunity to reduce false diagnosis and treatment failure by enhancing predictability at all stages. Mining external data in a much broader scope can enable modeling and simulation of treatment options in order to anticipate and de-risk them. The anticipative use of better disease definition and real world evidence data will provide meaningful medically relevant approaches which will place us in a much better position to execute successful precision medicine. Finally, this approach will also lead us to create new forms of precompetitive "open innovation" models. ${ }^{6}$

In the open network of MedBiomiX, ${ }^{7}$ we propose to set up a cross-functional and stepwise project driven initiatives to generate an accessible Translational data-Hub, starting in Hospitals at the very bedside, strengthening the portfolio across the network by increasing knowledge sharing. A small taskforce, led by medical experts, with selected key genomic and information technology stakeholders is being set up to prepare how we can find synergies and boost the use of "Actionable Computer Assisted Contextual Information Acquisition" (ACACIA).

As we want to align Big Data opportunities with our goals on a project basis, ACACIA will be run on selected projects, relying on the existing new IT-technologies deployed with Centers of Excellence in Translational Medicine.
Volume I Issue 3 - 2014

\author{
Patrice P Denefle \\ MedBiomiX Partners, Saint Maur FRANCE, France
}

Correspondence: Patrice P Denefle, MedBiomiX Partners, Saint Maur FRANCE, France, Email pdenefle@medbiomix.com

Received: July 10, 2014 | Published: August 01, 2014

In addition, Open source publications is a mandatory part of the transformation process, increasing the number of publications in MOJPB will contribute to the dissemination of new treatment algorythms and theranostics leading to improved patient health. Very few currently available online journals have such a rare opportunity to contribute directly to patients care through the publications of clinically relevant studies that have translational Medicine value. It is thus our pleasure to be part of the journal Editorial Board to continue its journey to deliver personalized medicine care to patients and to guide them towards informed treatment decisions.

\section{Acknowledgements}

None.

\section{Conflict of interest}

The author declares no conflict of interest.

\section{References}

1. http://www.libertyglobal.com/PDF/public-policy/The-Value-of-OurDigital-Identity.pdf

2. http://www.oracle.com/us/industries/health-sciences/omics-data-modelds-1506694.pdf

3. https://icgc.org

4. https://www.humanbrainproject.eu/fr

5. Suparna C, Jennifer RF, Michelle LM, et al. Big data, open science and the brain: lessons learned from genomics. Front Hum Neurosci. 2014.

6. Sean E, Chris LW, Mary PB, et al. Four disruptive strategies for removing drug discovery bottle necks. Drug Discovery Today. 2013;18(5-6):265271 .

7. http://www.medbiomix.com 